\title{
MiR-203 Determines Poor Outcome and Suppresses Tumor Growth by Targeting TBK1 in Osteosarcoma
}

\author{
Shiping Liu Peng Feng \\ Departments of Orthopedics, Yan'an People's Hospital, Yan'an, China
}

\section{Key Words}

MiR-203 • Osteosarcoma • Tumor suppressor • TBK1

\begin{abstract}
Background/Aims: Increasing evidence has shown that miR-203 plays important role in human cancer progression. However, little is known about the function of miR-203 in osteosarcoma (OS). Methods: The expression of miR-203 in OS tissues and cell lines were examined by qRTPCR. The biological role of miR-20 in OS cell proliferation was examined in vitro and in vivo. The targets of miR-203 were identified by a luciferase reporter gene assay. Results: miR-203 was down regulated in OS tissues and cell lines; decreased miR-203 was associated with a poor overall survival in OS patients. Restoration of miR-203 expression reduced cell growth in vitro and suppressed tumorigenicity in vivo. In contrast, inhibition of miR-203 stimulated OS cell growth both in vitro and in vivo. In addition, TANK binding kinase 1 (TBK1) was identified as a direct target of miR-203; overexpression of TBK1 partly reversed the suppressive effects of miR-203. Furthermore, TBK1 was found up-regulated and inversely correlated with miR203 in OS tissues. Conclusion: Taken together, these findings suggest that miR-203 acts as a tumor suppressor via regulation of TBK1 expression in OS progression, and miR-203 may be a promising therapeutic target for OS.
\end{abstract}

(C) 2015 The Author(s)

Published by S. Karger AG, Basel

\section{Introduction}

Osteosarcoma (OS) is one of the most common malignancies in children and young adults worldwide [1]. Despite the surgical techniques and chemotherapeutic treatments for OS have been significantly improved, $30 \%$ of children diagnosed with OS will not survive for more than five years [1-3]. Therefore, a comprehensive understanding of OS biology is required to optimize the diagnosis, therapy and prognosis prediction of this disease.

S. Liu and P. Feng contributed equally to this work.

Shiping Liu and Peng Feng

Departments of Orthopedics, Yan'an People's Hospital, No 57 Qilipu Street, Yan'an, Shaanxi 716000, (China)

E-Mail dr_spliu99@hotmail.com and E-Mail fengflowpeng@126.com 
MicroRNAs (miRNAs) are non-coding small RNAs of 18-22 nucleotides in length that regulate target genes expression at the post-transcriptional level through interaction with the 3' untranslated region (3'UTRs) of specific target mRNAs [4]. A large number of studies have proved that deregulation or dysfunction of miRNAs contributes to cancer development and miRNAs may act as tumor suppressors or oncogenes [5].

MiR-203 was originally detected in keratinocyte that promoted epidermal differentiation and was involved in the pathogenesis of psoriasis $[6,7]$, but later it was found to be ubiquitously expressed in various tissues and was associated with several human malignancies. Down regulation of miR-203 expression was found in several types of cancers, including gastric cancer [8], hepatocellular carcinoma (OS) [9], non-small cell lung cancer (NSCLC) [10], cervical cancer [11], esophageal cancer [12] as well as melanoma [13]. However, studies also showed that miR-203 was up regulated in breast cancer [14], colorectal cancer [15], ovarian cancer [16] and pancreatic adenocarcinoma [17]. Recently, increasing evidences suggest that miR-203 functions as a tumor suppressor in a tissue- or cell-lineage-specific manner by targeting multiple genes [18]. However, in contrast to other cancer types, little is known about the role of miR-203 in the pathogenesis of OS.

In the current study, we found that miR-203 was down regulated in OS tissues and cell lines, decreased miR-203 was associated with a poor overall survival in OS patients. Restoration of miR-203 expression reduced cell growth in vitro and suppressed tumorigenicity in vivo. In contrast, inhibition of miR-203 stimulated OS cell growth both in vitro and in vivo. In addition, TANK binding kinase 1 (TBK1) was identified as a direct target of miR-203; overexpression of TBK1 partly reversed the suppressive effects of miR-20. Furthermore, TBK1 was found up-regulated and inversely correlated with miR-203 in OS tissues. Taken together, our findings collectively suggest that miR-203 acts as a tumor suppressor via regulation of TBK1 expression and may thus be a promising therapeutic target for OS.

\section{Materials and Methods}

\section{Human specimens and cell culture}

60 paired fresh surgically resected osteosarcoma tumor tissues and adjacent non-tumor tissues were collected from the Departments of Orthopedics, Yan'an People's Hospital between November 2009 and November 2011. Specimens were gently washed with normal saline and flash-frozen in liquid nitrogen immediately after collection and stored at $-80^{\circ} \mathrm{C}$ until use. Both tumor and non-tumor samples were confirmed by pathological examination. The patients' characteristics at the original diagnosis are summarized in Table 1. This research was approved by the Research Ethics Committee of Yan'an People's Hospital. Human osteosarcoma cell lines (MG-63, U20S, HOS and Saos-2), human normal osteoblast cells NHOst were purchased from American Type Culture Collection (ATCC; Rockville, MD, USA). Cells were cultured in Dulbecco's modified Eagle's medium (Gibco BRL, Grand Island, NY) containing 10\% fetal bovine serum, penicillin $(100 \mathrm{U} / \mathrm{mL})$, and streptomycin $(100 \mu \mathrm{g} / \mathrm{mL})$ at $37^{\circ} \mathrm{C}$ in a humidified incubator containing $5 \% \mathrm{CO}_{2}$.

\section{RNA extraction and real-time quantitative PCR}

Total RNA was extracted from tissues and cells using miRNeasy Mini Kit (QIAGENE, Shanghai, China), according to the manufacturer's protocol. Total RNA concentration was assessed by measuring absorbance at $260 \mathrm{~nm}$ using a NanoDrop spectrophotometer (ND-1000, Thermo Scientific, Waltham, MA, USA). $2 \mu \mathrm{g}$ of total RNA was reversely transcribed using the PrimeScript RT reagent kit with gDNA Eraser (TaKaRa, Japan) and miRNA-specific stem-loop RT primer (Applied Biosystems, USA). Stem-loop RT primer for miR203 was: 5'- GTC GTA TCC AGT GCA GGG TCC GAG GTA TTC GCA CTG GAT ACG ACC TAG TGGTC-3'. Genespecific amplification was performed using ABI 7500 fast real-time PCR system (Applied Biosystems, Foster City, CA, USA) and SYBR Green PCR Master Mix (Applied Biosystems, Foster City, CA, USA) according to the manufacturer's instructions. The following gene-specific primers were used in this study: forward, 5'-GTA TCC AGT GCA GGG TCCGA-3' and reverse 5'-CGA CGG TGA AAT GTT TAG-3' for miR-203; forward, 5'-TGA AGA GGA GAC AAC AAC AAG-3' and reverse 5'- GTG CCA TAC AGA GAA ACA AAC-3' for TBK1; forward, 5'-AGA GCC

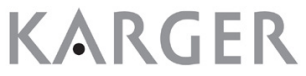




\section{Cellular Physiology Cell Physiol Biochem 2015;37:1956-1966

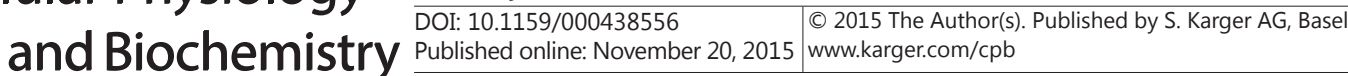 \\ Liu/Feng: MiR-203 Suppresses OS by Inhibiting TBK1}

TGT GGT GTCCG-3' and reverse, 5'-CAT CTT CAA AGC ACT TCCCT-3' for U6 small nuclear RNA; forward, 5'-CAT GTT CGT CAT GGG TGT GAA CCA3' and reverse, 5'-AGT GAT GGC ATG GAC TGT GGT CAT-3' for GAPDH. The relative expression level of miR-203 and TBK1 were normalized to that of internal control U6 or GAPDH using the comparative delta CT $\left(2^{-\Delta \Delta \mathrm{Ct}}\right)$ method. Each sample was analyzed in triplicate and the mean expression level was calculated.

\section{Cell Transfection}

The miR-203 mimics, miR-203 inhibitor and negative control (NC) were designed and synthesized by GenePharma (GenePharma, Shanghai, China). For transfection, $2 \times 10^{5}$ MG-63 and U2OS cells were seeded into 6-well plate in growth medium without serum and antibiotics at a density of $30-40 \%$ and incubated overnight, then transfected with miR-203 mimics, miR203 inhibitor or negative control (NC) using HiPerFect Transfection Reagent (Qiagen, Hilden, German) according to the manufacturer's protocol. The mixture was added to cells at a final concentration of $100 \mathrm{nM}$. After incubation in an environment with $5 \% \mathrm{CO}_{2}$ at $37^{\circ} \mathrm{C}$ for $4-6 \mathrm{~h}$, the serum- free medium was removed, and cells were maintained in DMEM containing 10\% FBS.
Table 1. Patient characteristics

\begin{tabular}{|c|c|c|c|c|}
\hline \multirow{2}{*}{ Characteristics } & \multirow{2}{*}{$\begin{array}{l}\text { Cases } \\
(60) \\
\end{array}$} & \multicolumn{2}{|c|}{ miR-203 expression } & \multirow{2}{*}{$P$ value } \\
\hline & & Low (43) & High (17) & \\
\hline \multicolumn{5}{|l|}{ Age (years) } \\
\hline$\leq 18$ & 33 & 23 & 10 & \multirow{2}{*}{0.708} \\
\hline$>18$ & 27 & 20 & 7 & \\
\hline \multicolumn{5}{|l|}{ Gender } \\
\hline Male & 32 & 24 & 8 & \multirow{2}{*}{0.540} \\
\hline Female & 28 & 19 & 9 & \\
\hline \multicolumn{5}{|l|}{ Tumor site } \\
\hline Femur/Tibia & 45 & 30 & 15 & \multirow{2}{*}{0.137} \\
\hline Others & 15 & 13 & 2 & \\
\hline \multicolumn{5}{|l|}{ Tumor stage } \\
\hline $\mathrm{I}+\mathrm{II}$ & 35 & 20 & 15 & \multirow{2}{*}{0.003} \\
\hline III & 25 & 23 & 2 & \\
\hline \multicolumn{5}{|c|}{ Tumor diameter $(\mathrm{cm})$} \\
\hline$\leq 6$ & 39 & 28 & 11 & \multirow{2}{*}{0.976} \\
\hline$>6$ & 21 & 15 & 6 & \\
\hline \multicolumn{5}{|l|}{ Distant metastasis } \\
\hline Yes & 24 & 21 & 3 & \multirow{2}{*}{0.026} \\
\hline No & 36 & 22 & 14 & \\
\hline \multicolumn{5}{|c|}{ Differentiation status } \\
\hline High & 34 & 26 & 8 & \multirow{2}{*}{0.345} \\
\hline Low & 26 & 17 & 9 & \\
\hline
\end{tabular}

\section{Cell proliferation assay}

After 24 hours of transfection, cells were harvested and seeded into 96-well plates at a density of $5 \times 10^{3}$ cells per well and cultured in an environment with $5 \% \mathrm{CO}_{2}$ at $37^{\circ} \mathrm{C}$ for $1,2,3,4$, days. $10 \mu \mathrm{l}$ of CCK-8 solution (Dojindo Laboratories, Kumamoto, Japan) was added into the culture medium in each well. After 1 hour incubation, OD values were read using a microplate reader (Bio-Tek Company, Winooski, VT, USA) at the 450-nm wavelength. Each time point was repeated in three wells and the experiment was independently performed for three times.

\section{Colony formation assay}

After 24 hours of transfection, cells were harvested and seeded into 24-well plates at a density of $5 \times$ $10^{2}$ cells per well and cultured in an environment with $5 \% \mathrm{CO}_{2}$ at $37^{\circ} \mathrm{C}$. During colony growth, the culture medium was replaced every 3 days. After 12 days, the plates were stained for the formation of cell colonies with crystal violet in $70 \%$ ethanol and counted under the microscope. The colony was counted only if it contained $>50$ cells. The experiment was independently performed for three times.

\section{Animal experiments}

Female 6-8 weeks old BALB/c nu/nu mice (Charles River Laboratories, Beijing, China) were housed in specific pathogen-free conditions. $2 \times 10^{6} \mathrm{MG}-63$ cells transfected with miR-203 agomir or agomir-negative control (GenePharma, China) were injected subcutaneously into the flank region of 6 week-old male nude mice ( $n=6$ per group). Tumor growth was monitored every 3 days and tumors were measured with fine digital calipers and tumor volume was calculated by the following formula: tumor volume $=0.5 \times$ width $^{2} \times$ length. After four weeks, the mice were sacrificed and tumor weighs were measured. All animal procedures were performed in accordance with protocols approved by the Institute Research Ethics Committee of Yan'an People's Hospital. 


\section{Cellular Physiology Cell Physiol Biochem 2015;37:1956-1966

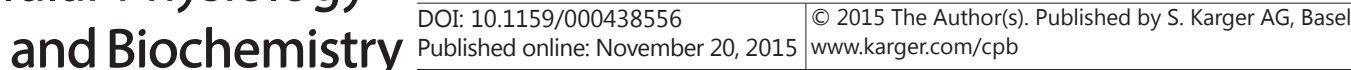 \\ Liu/Feng: MiR-203 Suppresses OS by Inhibiting TBK1}

Plasmid construction and luciferase reporter assay

The 3'-UTR of TBK1 containing the putative miR-203 binding sequence was amplified by PCR and cloned downstream of the firefly luciferase gene in the pMIR-Report vector (Promega, Madison, WI, USA). Mutation in the miR-203 seed regions of the TBK1 3'UTR were generated using QuikChang Multisitedirected mutagenesis kit (Promega, Fitchburg, WI, USA). The mutated TBK1 3'-UTR fragment was cloned into the pMIR-Report vector to develop the pMIR-TBK1-3'UTR-mut vector. For the luciferase assay in the OS cells, MG-63 or U2OS were co-transfected with 50ng/well TBK1-3'UTR or TBK1 3'UTR-mut, and $50 \mathrm{nM} /$ well miR-203 mimic or scrambled microRNA negative control using Lipofectamine 2000 reagent (Invitrogen, Carlsbad, CA, USA). Cells were collected after $48 \mathrm{~h}$ for assay using the Dual Luciferase reporter assay system (Promega, Fitchburg, WI, USA).

\section{Western blotting}

The cells were lysed in RIPA buffer with protease inhibitor at $72 \mathrm{~h}$ post-transfection. Total protein was separated by $10 \%$ SDS-PAGE, transferred onto polyvinylidene fluoride membranes. The primary antibodies to TBK1 (1:500; Santa Cruz, CA, USA) and $\beta$-actin (1:2000; Santa Cruz, CA, USA) were incubated with the blot overnight at $4^{\circ} \mathrm{C}$. After being extensively washed with PBS containing $0.1 \%$ Triton X-100, the membranes were incubated with HRP-conjugated goat anti-rabbit antibody for $30 \mathrm{~min}$ at room temperature. The bands were visualized using the ECL system (Millipore, Billerica, WI, USA).

\section{Statistical analysis}

Statistical Package of the Social Sciences 19.0 for Windows (SPSS, Chicago, IL, USA) was used for statistical analyses. The survival rate of OS patients was calculated using Kaplan-Meier survival analysis. Differences between experimental groups were assessed using the two-tailed unpaired Student's t test. The relationship between miR-203 and TBK1 expression was assessed using Spearman's correlation analysis. $P<0.05$ was considered as statistical significance.

\section{Results}

MiR-203 is down regulated in human OS tissues and cell lines

To understand the role of miR-203 in OS, miR-203 expression was examined by qRTPCR in 60 OS tissues and the corresponding normal bone tissues. The results showed that miR-203 was down regulated in the majority of OS tissues (Fig. 1A). Moreover, miR-203 was down regulated in four human OS cell lines MG-63, U2OS, HOS and Saos-2 compared with normal osteoblastic cell lines NHOst (Fig. 1B). Furthermore, clinical results showed that miR-203 expression was inversely correlated with tumor stage and distant metastasis $(P=$ 0.003 and $P=0.026$, respectively, Table 1). Patients with lower expression of miR-203 had a poor prognosis than those with high expression of miR-203 (Fig. 1C). These results suggest that miR-203 plays an important role in OS development.

\section{MiR-203 suppresses OS growth in vitro and in vivo}

Next, we investigated the effects of miR-203 on OS cell growth by overexpression or inhibition of miR-203 expression in MG-63 and U2OS cells (Fig. 2A). As shown in Fig. 2B, overexpression of miR-203 significantly inhibited MG-63 cell growth. In contrast, inhibition of miR-203 stimulated MG-63 cell growth. In addition, colony formation assay showed that overexpression of miR-203 repressed MG-63 colony formation, whereas inhibition of miR-203 promoted MG-63 colony formation compared to the control (Fig. 2C). We further confirmed these results using another OS cell line U2OS (Fig. 2D and E).

To determine if this effect applied in vivo, we confirmed these in vitro results using a xenograft model. MG-63 cells transfected with agomiR-203 or negative control (agomiRNC) were subcutaneously injected into the flank region of nude mice. As shown in Fig. 2F, overexpression of miR-203 inhibited tumor growth compared to the control group, the tumor weights in agomiR-203 group were significantly decreased compared to the agomiRNC group (Fig. 2G). Collectively, our data showed that miR-203 suppresses OS cell growth in vitro and in vivo. 


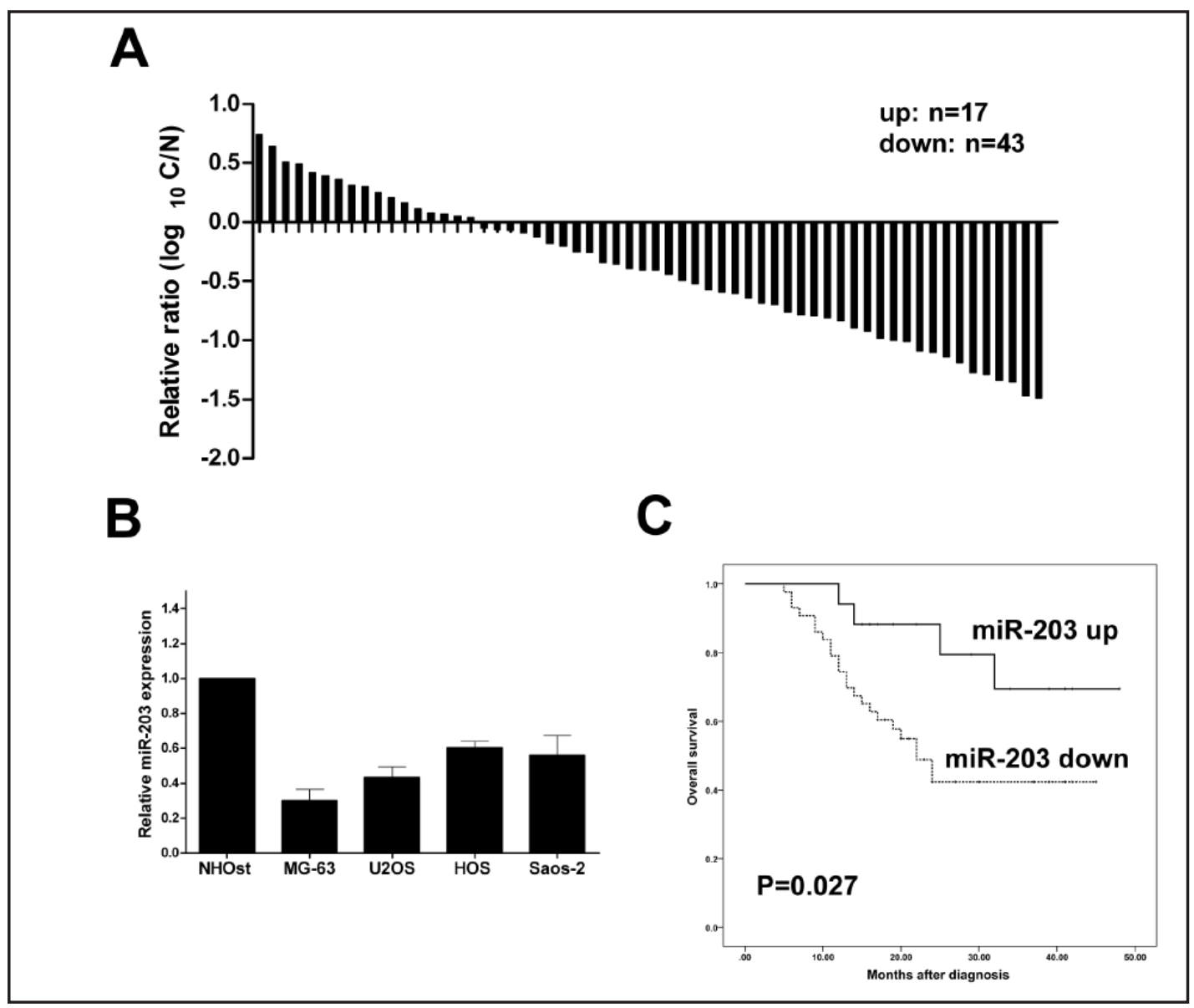

Fig. 1. MiR-203 is down regulated in human OS tissues and cell lines. (A) The expression of miR-203 in 60 paired of OS samples and their corresponding normal bone tissues was detected using qRT-PCR. (B) miR203 expression in human OS cell lines (MG-63, U2OS, HOS and Saos-2), and normal osteoblastic cell line NHOst was detected by qRT-PCR. (C) Kaplan-Meier curve for OS patients classified as high or low miR-203 expression, the $P$-value was calculated using the log-rank test.

TBK1 is a direct target of miR-203

Bioinformatics research was performed to find potential targets of miR-203 using Targetscan and miRanda. As shown in Fig. 3A, TBK1 was identified as a potential target gene of miR-203, with the predicted binding site at the base from positions 526 to 531 . TBK1 is a noncanonical I $\kappa$ B kinase (IKK) family member and plays a vital role in cell survival and tumor growth. To validate whether the 3'-UTR of TBK1 is a functional target of miR-203, a dual-luciferase reporter system was employed. We cloned 3'UTR sequences containing wild type or mutated binding site of miR-203 into the pMIR-Report vector, respectively, and co-transfected with the miR-203 mimics or NC into OS cells. Data from the luciferase assay showed that overexpression of miR-203 remarkably suppressed the luciferase activity of the reporter gene with the wild-type construct but not with the mutant TBK1 3'UTR construct in OS cells (Fig. 3B and C). In addition, overexpression of miR-203 resulted in a reduction of TBK1 mRNA and protein expression in OS cells (Fig. 3D and E). Therefore, our results suggest that TBK1 is a direct target of miR-203 in OS cells.

Restoration of TBK1 rescues the suppressive effects of miR-203 on OS cell growth

To further confirm TBK1 is a direct target of miR-203, we co-transfected MG-63 cells with miR-203 mimics and TBK1 plasmid with or without 3'UTR. As shown in Fig. 4A, miR- 


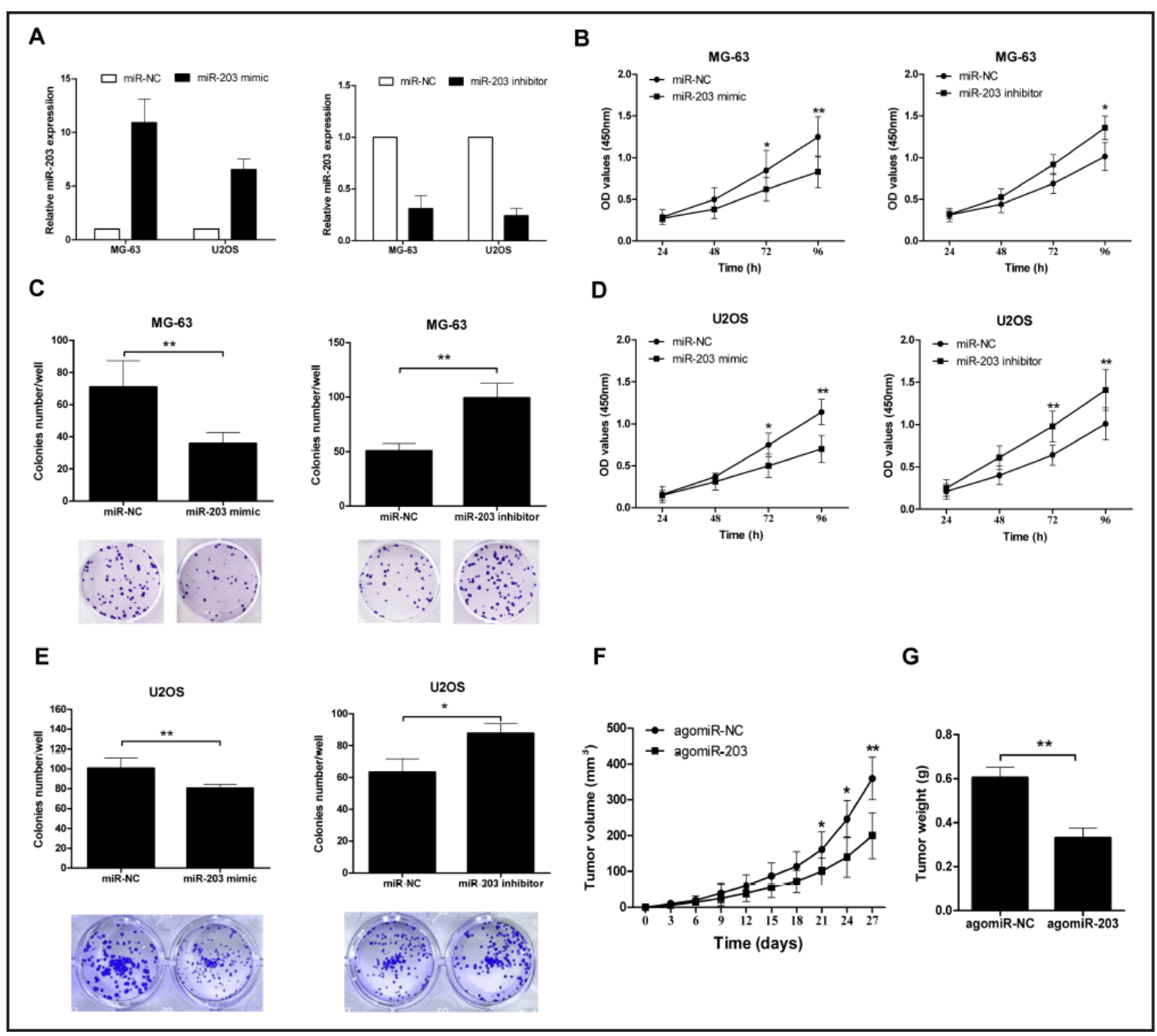

Fig. 2. MiR-203 suppresses OS growth in vitro and in vivo. (A) MG-63 and U20S cells were transfected with hsa-miR-203 mimics, miR-203 inhibitor or negative control (NC), respectively. The expression of miR-203 was confirmed by qRT-PCR in both MG-63 and U20S cell lines. (B and D) Overexpression of miR-203 inhibits MG-63 or U20S proliferation, whereas inhibition of miR-203 stimulated MG-63 or U2OS proliferation compared to the controls. Cell proliferation was measured by a CCK-8 assay at the indicated time. (C and E) Overexpression of miR-203 inhibited colony formation, whereas inhibition of miR-203 stimulated colony formation compared to the control in MG-63 or US20S cells. Colony formation was measured 12 days after transfection. ( $F$ and G) Overexpression of miR-203 inhibited tumor growth, whereas inhibition of miR-203 stimulated tumor growth in a xenograft model. MG-63 cells transfected with agomiR-203 or negative control (agomiR-NC) were subcutaneously injected into the flank region of nude mice. Tumor volumes were measured every 3 days, and the tumor weights were measured on day 28. Data shown are mean \pm SD from three independent experiments. ${ }^{*} P<0.05,{ }^{* *} P<0.01$.

203-mediated TBK1 down regulation was restored following co-transfection with TBK1 plasmid without 3'UTR. Additionally, the inhibitory role of miR-203 in cell growth and colony formation was rescued under the condition of overexpression of TBK1 without 3'UTR (Fig. 4B and C). Taken together, these data suggest that TBK1 is a direct and functional downstream target of miR-203 in OS cells.

TBK1 is up-regulated and inversely correlated with miR-203 in OS tissues

Lastly, we investigated whether TBK1 was up regulated in OS tissues and associated with miR-203 expression. As shown in Fig. 5A and B, TBK1 was found up-regulated in the 
Fig. 3. MiR-203

directly targeted TBK1 in OS cells.

(A) Sequence alignment of wild-type (WT) and mutated (Mut) putative miR-203-binding sites in the 3'-UTR of TBK1. (B and C) Relative luciferase activities of plasmids carrying WT or mutant TBK1 3'UTR in MG-63 and U2OS cells co-transfected with miR-203 mimics or miR-NC. (D and E) The mRNA and protein levels of TBK1 in MG-63 and U20S cells were examined by western blot after co-transfection. Data shown are mean \pm SD from three independent experiments. ${ }^{*} P<0.05,{ }^{* *} P<0.01$, ${ }^{* * *} P<0.001$.

\section{A \\ TBK1 3'UTR WT \\ (526-531) \\ hsa-miR-203}

TBK1 3' UTR mutant

B

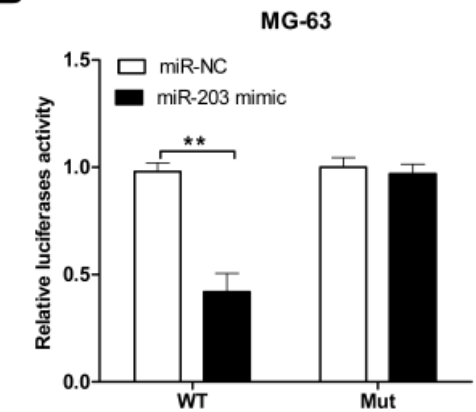

D

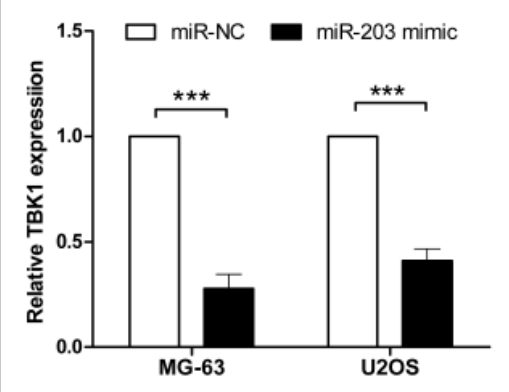

C
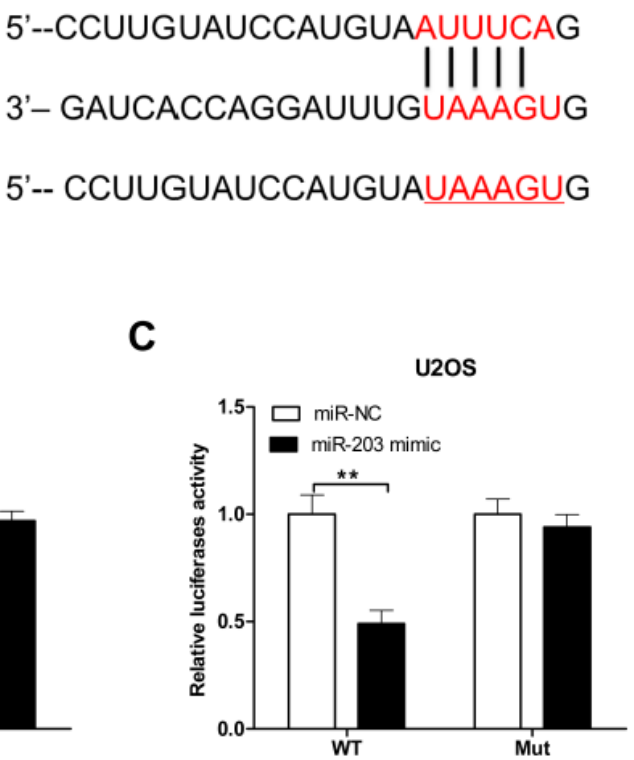

5'-- CCUUGUAUCCAUGUAUAAAGUG

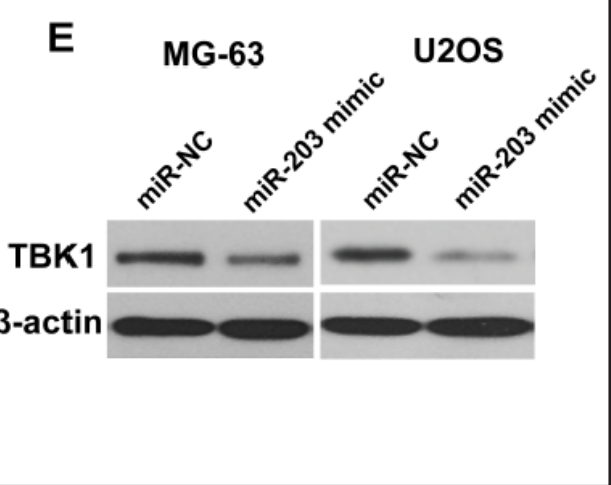

majority of OS tissues compared to matched normal tissues. In addition, correlation analyses revealed that there was a significant inverse correlation between miR-203 and TBK1 expression levels in OS tissues (Fig. 5C).

\section{Discussion}

In this study, we investigated the biological role of miR-203 in the progression of OS. We found significant down-regulation of miR-203 in OS tissues compared with matched normal bone tissues. Additionally, functional experiments demonstrated that miR-203 suppressed cell growth in vitro and inhibited tumor growth in vivo through targeting the TBK1. Our findings suggest that miR-203 acts as a novel tumor suppressor by targeting TBK1 in OS. Our work may be the first time to reveal the role of miR-203 in OS.

Recently, accumulating evidence indicates that miR-203 is down-regulated in various tumors, and functions as a tumor suppressor in a tissue- or cell-lineage-specific manner by targeting multiple genes. Wan et al. reported that miR-203 functions as a tumor suppressor in OS, and regulates cell proliferation and metastasis by directly targeting oncogene ADAM9 and oncogenic long non-coding RNA HULC [9]. Moreover, down-regulation of miR-203 induced by Helicobacter pylori infection promoted the proliferation and invasion of gastric cancer by 


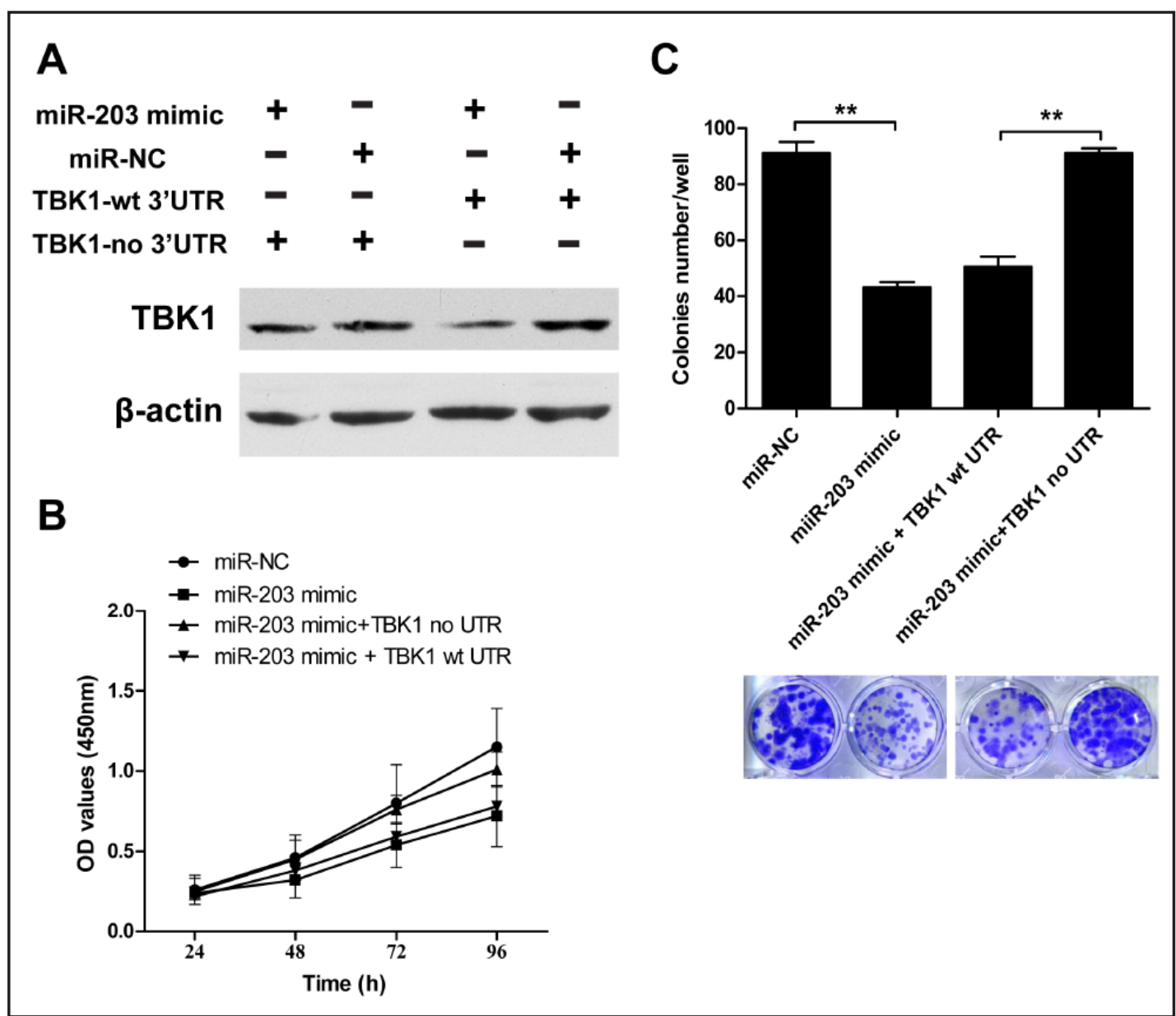

Fig. 4. Restoration of TBK1 rescues the suppressive effects of miR-203 on OS cell growth. (A) MG-63 cells were co-transfected with miR-203 mimics or miR-NC and TBK1 plasmid with or without 3'UTR, the expression levels of TBK1 were examined by western blot. The effect of miR-203 on cell proliferation (B), and colony formation (C) was rescued under the condition of overexpression of TBK1 without 3'UTR. Data shown are mean \pm SD from three independent experiments. ${ }^{* *} P<0.01$.

targeting CASK [8]. Zhang et al. found that miR-203 suppressed tumor growth and invasion and down-regulated MiR-21 expression through repressing Ran in esophageal cancer [12]. However, Naoki reported that miR-203 was highly expressed in pancreatic adenocarcinoma, and miR-203 overexpression indicated poor prognosis in pancreatic adenocarcinoma patients who underwent pancreatectomy [17]. These findings suggest the complicated role of miR-203 in cancer progression, and the different role of miR-203 was possibly due to the fact that miRNAs could down-regulate numerous targets, including oncogenes and tumor suppressors. Therefore, the function of miR-203 in carcinogenesis and the development of tumors should be clarified in the future. In this study, we found that miR-203 suppressed OS cell growth and colony formation in vitro and inhibits OS growth in vivo, suggesting a tumor suppressor role of miR-203 in OS.

To determine how miR-203 acts as a tumor suppressor, we screened the target genes of miR-203 using bioinformatics analysis. TBK1 was selected as a potential target gene of miR-203 based on its particular functions and expression patterns. It had not been reported whether miR-203 could directly target TBK1 in OS. TBK1 belongs to the noncanonical IкB kinase (IKK) family, and served as an activator of the oncogenic AKT kinase and an 


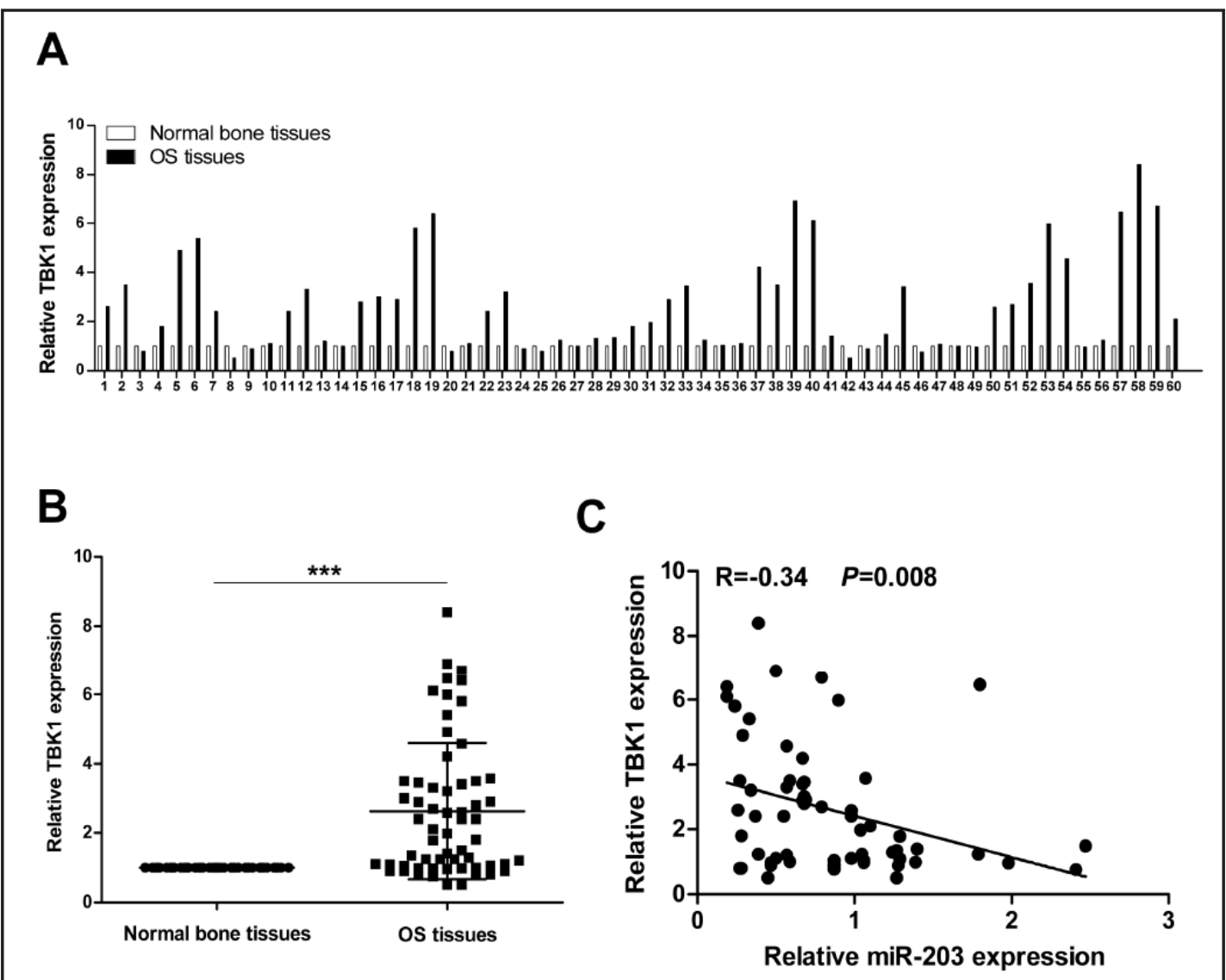

Fig. 5. TBK1 was up-regulated in OS tissues and inversely correlated with miR-203. (A and B) The expression of TBK1 in 60 pairs of OS tissues and matched normal bone tissues was examined by qRT-PCR. (C) The correlation between miR-203 and TBK1 mRNA levels in OS tissues was analyzed using Spearman's correlation analysis. Data shown are mean \pm SD. ${ }^{* * *} P<0.001$.

activator of the KRAS driven cancer $[19,20]$. In addition, it has been identified as a target of several miRNAs involved in carcinogenesis and tumor progression. For example, TBK1 was identifies as a downstream effector of the miR-200c in human lung cancer cells [21]. Inhibition of TBK1 attenuates radiation-induced epithelial-mesenchymal transition via activation of GSK-3 $\beta$ and repression of ZEB1 in lung cancer [22]. More recently, in pancreatic ductal adenocarcinoma (PDAC), miR-429 inhibited PDAC cell lines growth by targeting TBK1 [23]. In our study, we verified TBK1 as a direct target of miR-203 using luciferase reporter gene assay, overexpression of miR-203 could repress both the mRNA and protein levels of TBK1. Moreover, the inhibitory effects of miR-203 on cell proliferation and colony formation were rescued under the condition of overexpression of TBK1. Additionally, we found that the expression of TBK1 was up-regulated in OS tissues, and miR-203 levels were inversely correlated with TBK1 expression in OS tissues. These data suggest that TBK1 is a direct and functional target of miR-203 in OS cells. However, the role of TBK1 in OS requires further investigation.

In summary, we combined clinical and experimental studies to establish a critical role for miR-203 in OS. We found low miR-203 level and high TBK1 level in OS promoted tumor cell growth, which contributed to poor survival for patients with OS. Our work led to the identification of a novel functional pathway controlled by miR-203 and its direct targets TBK1 in OS. Therefore, miR-203 may represent a novel therapeutic target for OS treatment. 


\section{Cellular Physiology Cell Physiol Biochem 2015;37:1956-1966

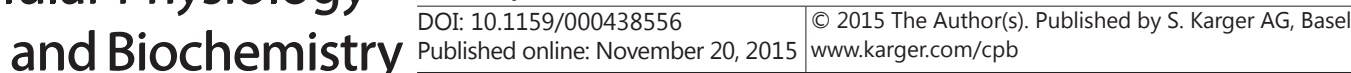 \\ Liu/Feng: MiR-203 Suppresses OS by Inhibiting TBK1}

\section{Acknowledgments}

This project was supported by grants from the Natural Science Foundation of Shannxi Province (No.2012k14-08-09).

\section{Disclosure Statement}

The authors have no conflict of interest.

\section{References}

1 Luetke A, Meyers PA, Lewis I, Juergens H: Osteosarcoma treatment - where do we stand? A state of the art review. Cancer Treat Rev 2014;40:523-532.

2 Benjamin RS: Osteosarcoma: better treatment through better trial design. Lancet Oncol 2015;16:12-13.

3 Li F, Li S, Cheng T: TGF-beta1 promotes osteosarcoma cell migration and invasion through the miR-143versican pathway. Cell Physiol Biochem 2014;34:2169-2179.

4 Bartel DP: MicroRNAs: genomics, biogenesis, mechanism, and function. Cell 2004;116:281-297.

5 Berindan-Neagoe I, Monroig Pdel C, Pasculli B, Calin GA: MicroRNAome genome: a treasure for cancer diagnosis and therapy. CA Cancer J Clin 2014;64:311-336.

6 Sonkoly E, Wei T, Janson PC, Saaf A, Lundeberg L, Tengvall-Linder M, Norstedt G, Alenius H, Homey B, Scheynius A, Stahle M, Pivarcsi A: MicroRNAs: novel regulators involved in the pathogenesis of psoriasis? PLoS One 2007;2:e610.

7 Yi R, Poy MN, Stoffel M, Fuchs E: A skin microRNA promotes differentiation by repressing 'stemness'. Nature 2008;452:225-229.

8 Zhou X, Xu G, Yin C, Jin W, Zhang G: Down-regulation of miR-203 induced by Helicobacter pylori infection promotes the proliferation and invasion of gastric cancer by targeting CASK. Oncotarget 2014;5:1163111640.

9 Wan D, Shen S, Fu S, Shen C, Wu J, Wang S, Xie W, Chen B, A L, Guo Y, Zheng D, Zhi Q Peng B: miR-203 suppresses the proliferation and metastasis of hepatocellular carcinoma by targeting oncogene ADAM9 and oncogenic long non-coding RNA HULC. Anticancer Agents Med Chem 2015: DOI 10.2174/1871520615 666150716105955.

10 Tang R, Zhong T, Dang Y, Zhang X, Li P, Chen G: Association between downexpression of MiR-203 and poor prognosis in non-small cell lung cancer patients. Clin Transl Oncol DOI:10.1007/s12094-015-1377-9

11 Zhu X, Er K, Mao C, Yan Q, Xu H, Zhang Y, Zhu J, Cui F, Zhao W, Shi H: miR-203 suppresses tumor growth and angiogenesis by targeting VEGFA in cervical cancer. Cell Physiol Biochem 2013;32:64-73.

12 Zhang F, Yang Z, Cao M, Xu Y, Li J, Chen X, Gao Z, Xin J, Zhou S, Zhou Z, Yang Y, Sheng W, Zeng Y: MiR-203 suppresses tumor growth and invasion and down-regulates MiR-21 expression through repressing Ran in esophageal cancer. Cancer Lett 2014;342:121-129.

13 Chang X, Sun Y, Han S, Zhu W, Zhang H, Lian S: MiR-203 inhibits melanoma invasive and proliferative abilities by targeting the polycomb group gene BMI1. Biochem Biophys Res Commun 2015;456:361-366.

14 Iorio MV, Ferracin M, Liu CG, Veronese A, Spizzo R, Sabbioni S, Magri E, Pedriali M, Fabbri M, Campiglio M, Menard S, Palazzo JP, Rosenberg A, Musiani P, Volinia S, Nenci I, Calin GA, Querzoli P, Negrini M, Croce CM: MicroRNA gene expression deregulation in human breast cancer. Cancer Res 2005;65:7065-7070.

15 Bandres E, Cubedo E, Agirre X, Malumbres R, Zarate R, Ramirez N, Abajo A, Navarro A, Moreno I, Monzo M, Garcia-Foncillas J: Identification by Real-time PCR of 13 mature microRNAs differentially expressed in colorectal cancer and non-tumoral tissues. Mol Cancer 2006;5:29.

16 Iorio MV, Visone R, Di Leva G, Donati V, Petrocca F, Casalini P, Taccioli C, Volinia S, Liu CG, Alder H, Calin GA, Menard S, Croce CM: MicroRNA signatures in human ovarian cancer. Cancer Res 2007;67:8699-8707.

17 Ikenaga N, Ohuchida K, Mizumoto K, Yu J, Kayashima T, Sakai H, Fujita H, Nakata K, Tanaka M: MicroRNA-203 expression as a new prognostic marker of pancreatic adenocarcinoma. Ann Surg Oncol 2010;17:3120-3128. 


\section{Cellular Physiology Cell Physiol Biochem 2015;37:1956-1966

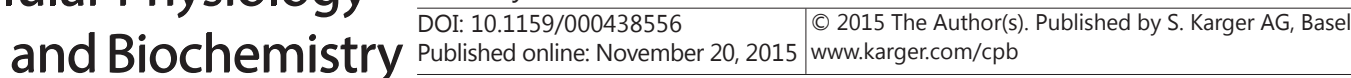

Liu/Feng: MiR-203 Suppresses OS by Inhibiting TBK1

18 Michel CI, Malumbres M: microRNA-203: Tumor Suppression and Beyond. Microrna 2013;2:118-126.

19 Barbie DA, Tamayo P, Boehm JS, Kim SY, Moody SE, Dunn IF, Schinzel AC, Sandy P, Meylan E, Scholl C, Frohling S, Chan EM, Sos ML, Michel K, Mermel C, Silver SJ, Weir BA, Reiling JH, Sheng Q Gupta PB, Wadlow RC, Le H, Hoersch S, Wittner BS, Ramaswamy S, Livingston DM, Sabatini DM, Meyerson M, Thomas RK, Lander ES, Mesirov JP, Root DE, Gilliland DG, Jacks T, Hahn WC: Systematic RNA interference reveals that oncogenic KRAS-driven cancers require TBK1. Nature 2009;462:108-112.

20 Ou YH, Torres M, Ram R, Formstecher E, Roland C, Cheng T, Brekken R, Wurz R, Tasker A, Polverino T, Tan SL, White MA: TBK1 directly engages Akt/PKB survival signaling to support oncogenic transformation. Mol Cell 2011;41:458-470.

21 Lin J, Liu C, Gao F, Mitchel RE, Zhao L, Yang Y, Lei J, Cai J: miR-200c enhances radiosensitivity of human breast cancer cells. J Cell Biochem 2013;114:606-615.

22 Liu W, Huang YJ, Liu C, Yang YY, Liu H, Cui JG, Cheng Y, Gao F, Cai JM, Li BL: Inhibition of TBK1 attenuates radiation-induced epithelial-mesenchymal transition of A549 human lung cancer cells via activation of GSK-3beta and repression of ZEB1. Lab Invest 2014;94:362-370.

23 Song B, Zheng K, Ma H, Liu A, Jing W, Shao C, Li G, Jin G: miR-429 determines poor outcome and inhibits pancreatic ductal adenocarcinoma growth by targeting TBK1. Cell Physiol Biochem 2015;35:1846-1856. 